\title{
Eine Psychotherapie ist immer nur so gut wie ihre Diagnostik
}

\author{
Ulrike Ehlert \\ Klinische Psychologie und Psychotherapie, Universität Zürich, Schweiz
}

Es steht außer Zweifel, dass verhaltenstherapeutische Interventionen bei einem breiten Spektrum psychischer Störungen und körperlicher Erkrankungen zu Beschwerdefreiheit oder Symptomreduktionen führen oder zumindest die Lebensqualität des erkrankten Menschen verbessern. Diese Erfahrungen machen nicht nur praktizierende Therapeutinnen und Therapeuten, es werden auch nahezu täglich neue Untersuchungen zur Wirksamkeit psychotherapeutischer Interventionen publiziert [vgl. Helbig et al., 2007].

Häufig werden Therapiestudien jedoch dafür kritisiert, dass die darin untersuchten Patientinnen oder Patienten «handverlesen» seien und die (zumeist) positiven Resultate nur durch eine starke Selektion der Studienteilnehmer und -teilnehmerinnen anhand hochspezifischer Ein- und Ausschlusskriterien erreicht worden seien. An dieser Kritik ist eine ganze Menge Wahrheit. Therapiestudien sind so angelegt, dass eine bestimmte Maßnahme, z.B. eine kognitiv-verhaltenstherapeutische Gruppenintervention für Frauen mit Wechseljahresbeschwerden, bezüglich ihrer Wirksamkeit im Vergleich zu Kontrollbedingungen geprüft werden kann [vgl. Bültzingslöwen et al., 2006]. Dazu müssen möglichst viele Unwägbarkeiten (konfundierende Variablen), die sich ebenfalls auf das Therapieergebnis auswirken könnten, minimiert werden. $\mathrm{Zu}$ dieser Reduktion von Unwägbarkeiten gehört es auch, dass möglichst eindeutige Diagnosen bei den zu behandelnden Patienten vorliegen. Die psychotherapeutischen Interventionen, die beispielsweise in Lehrbüchern im Rahmen von Fallvignetten vorgestellt werden, werden daher meist bei Patientinnen und Patienten mit genau der Diagnose angewendet, für die eine bestimmte Therapie beschrieben wird.

Die alltägliche psychotherapeutische Erfahrung zeigt jedoch, dass sich bei Patientinnen und Patienten üblicherweise eine Vielzahl konfundierender Merkmale finden (z.B. Einnahme unterschiedlicher Medikamente, mehrere Diagnosen etc.) und dass die beschriebenen Therapieansätze nur für einen Teil der
Symptome anwendbar sind (vgl. z.B. Leo [2005] für den Zusammenhang zwischen Schmerz und Depression). In einem großen Forschungsprojekt unserer Arbeitsgruppe zu psychotherapeutischer Kurzzeitintervention im Allgemeinkrankenhaus an über 1000 untersuchten Patientinnen und Patienten fanden sich zwar bei etwa einem Drittel davon depressive Symptome, jedoch reduzierten sich die Fallzahlen für eine wissenschaftliche Evaluation der psychotherapeutischen Intervention bei depressiven Patienten auf jeweils 30 Patientinnen und Patienten für die diagnostischen Hauptgruppen der Depression (Major Depression Einzelepisode, Major Depression rezidivierend oder Anpassungsstörung) [Ehlert, 1998]; die anderen Patientinnen und Patienten mit depressiven Symptomen hatten entweder komorbide psychische Störungen (z.B. Alkoholismus, Posttraumatische Belastungsstörung), waren zu jung oder zu alt für das intendierte Therapieprogramm oder wurden auch pharmakologisch behandelt und waren daher nicht vergleichbar mit anderen depressiven Patientinnen oder Patienten.

Was lässt sich aus dieser Erfahrung schließen? Eine psychotherapeutische Intervention wirkt nur dann, wenn das gewählte Vorgehen zu den spezifischen Merkmalen und Symptomen der Patientin bzw. des Patienten «passt». Diese Passung lässt sich dann erreichen, wenn eine umfassende Diagnostik erfolgt, so dass möglichst viele therapierelevante Aspekte bekannt sind. Hierfür steht neben den diagnostischen Grundtechniken der umfassenden Anamnese und der Verhaltensanalyse ein äußerst differenziertes Repertoire von Interviews, Selbst- und Fremdbeurteilungsverfahren sowie Selbst- und Fremdbeobachtungsverfahren zur Verfügung. Symptom-Monitoring-Systeme und elektronische Tagebücher lassen sich heute aufgrund der gerätetechnischen Möglichkeiten zuverlässig im therapeutischen Alltag einsetzen [vgl. Fahrenberg et al., 2007]. Darüber hinaus können verschiedene psychobiologische Parameter erhoben werden; von Aktivitätsmessungen

\begin{tabular}{ll}
\hline KARGER & @ 2007 S. Karger GmbH, Freiburg \\
Fax +497614520714 & Accessible online at: \\
$\begin{array}{l}\text { E-mail Information@Karger.de } \\
\text { www.karger.com }\end{array}$ & www.karger.com/ver \\
&
\end{tabular}

Prof. Dr. rer. nat. Ulrike Ehlert 
mittels Actiwatch ${ }^{\circledR}$ (med-NATIC; München, Deutschland) oder Herzratenmessungen mittels entsprechender Fitnessuhren bis hin zu endokrinologischen oder immunologischen Maßen, wie sie von Manfred Schedlowski für den Bereich der Psychoneuroimmunologie skizziert wurden [2007].

Eine umfassende Diagnostik setzt jedoch voraus, dass wir uns über diagnostische Verfahren informieren und bereit sind, neben dem einmal zusammengestellten und bewährten Standardrepertoire von Fragebogen oder Interviews neue Erhebungsinstrumente, ihre Testgüte, aber auch ihre Alltagstauglichkeit und den möglichen Erkenntnisgewinn auszuprobieren. Wird die Differentialdiagnostik durch «neue» Messinstrumente verbessert und damit die Behandlung von Patientinnen und Patienten «passgenauer» gemacht, profitieren nicht nur die zu Behandelnden, sondern auch die Therapeutinnen und Therapeuten. Denn letztgenannte definieren ihre persönliche, berufsbezogene Zufriedenheit sicherlich auch darüber, wie gut ihre Annahmen über ihre Patientinnen und Patienten zu den im Verlauf der Therapie gewählten Strategien und dem daraus resultierenden Therapieerfolg passen.
Daher gilt: Eine Psychotherapie ist nur so gut wie ihre Diagnostik. Nur eine differenzierte Beschreibung und Beurteilung erlaubt eine gute Indikationsstellung und führt dazu, dass bereits zu Beginn der Therapie realistisch eingeschätzt werden kann, ob es sich um eine Bilder- bzw. Lehrbuchtherapie oder ein langwierigeres, hochkomplexes Therapiegeschehen handeln wird, bei dem auf beiden Seiten des therapeutischen Geschehens viel Mut und Ausdauer gebraucht wird.

\section{Literatur}

Bültzingslöwen von K, Pfeifer M, Kröner-Herwig B: Ein kognitiv-verhaltenstherapeutisches Gruppentraining für Frauen mit Wechseljahresbeschwerden - Ergebnisse einer kontrollierten Gruppenstudie. Verhaltenstherapie 2006;16:184-192.

Ehlert U: Psychologie im Allgemeinkrankenhaus. Bern, Huber, 1998.

Fahrenberg J, Myrtek M, Pawlik K, Perrez M: Ambulantes Assessment - Verhalten im Alltagskontext erfassen. Psychol Rundsch 1988;58:12-23.

Helbig S, Hoyer J: Hilft wenig viel? Eine Minimalintervention für Patienten während der Wartezeit auf ambulante Verhaltenstherapie. Verhaltenstherapie 2007;17: 109-115.

Leo RJ: Chronic pain and comorbid depression. Curr Treat Opt Neurology 2005; 7:403-412.

Schedlowski M: «Gezielte Verhaltensinterventionsprogramme können das biochemische Netzwerk im Körper beeinflussen». Verhaltenstherapie 2007;17:129-131. 